\title{
Performance of Firth-and $\log F$-type penalized methods in risk prediction for small or sparse binary data
}

\author{
M. Shafiqur Rahman* and Mahbuba Sultana
}

\begin{abstract}
Background: When developing risk models for binary data with small or sparse data sets, the standard maximum likelihood estimation (MLE) based logistic regression faces several problems including biased or infinite estimate of the regression coefficient and frequent convergence failure of the likelihood due to separation. The problem of separation occurs commonly even if sample size is large but there is sufficient number of strong predictors. In the presence of separation, even if one develops the model, it produces overfitted model with poor predictive performance. Firth-and log F-type penalized regression methods are popular alternative to MLE, particularly for solving separation-problem. Despite the attractive advantages, their use in risk prediction is very limited. This paper evaluated these methods in risk prediction in comparison with MLE and other commonly used penalized methods such as ridge.

Methods: The predictive performance of the methods was evaluated through assessing calibration, discrimination and overall predictive performance using an extensive simulation study. Further an illustration of the methods were provided using a real data example with low prevalence of outcome.

Results: The MLE showed poor performance in risk prediction in small or sparse data sets. All penalized methods offered some improvements in calibration, discrimination and overall predictive performance. Although the Firth-and $\log F$-type methods showed almost equal amount of improvement, Firth-type penalization produces some bias in the average predicted probability, and the amount of bias is even larger than that produced by MLE. Of the $\log F(1,1)$ and $\log F(2,2)$ penalization, $\log F(2,2)$ provides slight bias in the estimate of regression coefficient of binary predictor and $\log F(1,1)$ performed better in all aspects. Similarly, ridge performed well in discrimination and overall predictive performance but it often produces underfitted model and has high rate of convergence failure (even the rate is higher than that for MLE), probably due to the separation problem.
\end{abstract}

Conclusions: The log F-type penalized method, particularly log $F(1,1)$ could be used in practice when developing risk model for small or sparse data sets.

Keywords: Prediction model, Separation, Performance measures, Overfitting

\section{Background}

In many areas of clinical research, risk models for binary data are usually developed in the maximum-likelihood (ML) based logistic regression framework to predict the risk of a patient's future health status such as death or illness $[1,2]$. For example, in cardiology, models may be developed to predict the risk of having cardiovascular disease. Predictions based on these models are useful

*Correspondence: shafiq@isrt.ac.bd

Institute of Statistical Research and Training, University of Dhaka, Dhaka, Bangladesh to both doctor and patient in making joint decision on future course of treatment. However, before using these models in risk prediction it is essential to assess their predictive performance using data other than that used to develop the models, which is termed as 'validation' [3, 4]. A good risk model is expected to demonstrate good calibration (accuracy of prediction) and discrimination (the ability of model to distinguish between low-and-high risk patients) in new dataset. A risk model that perform well in development data (that used to fit the model called 'training' set) may not perform similar to the validation 
data (that used to validate the model called 'test' set). One of the main reasons for not performing well in test data is model overfitting which causes too high prediction for high risk patients and too low for low risk patients. The overfitting occurs frequently when the number of events in training data is lower than the number of risk factors. After employing expert knowledge even if one fits the model with reduced the number of predictors, the ratio of the number of event to the number of predictors (EPV) often very low. However, as a rule of thumb, it has been suggested in literature that the risk model performs well when EPV is at least 10 [5]. Although the choice of this cut-off has some criticisms [6] for not being based on scientific reasoning except empirical evidence, it is found useful for quantifying the amount of information in the data relative to model complexity $[7,8]$. However, the requirement of minimum EPV is often difficult to achieve when the risk models develop for low-dimensional data with rare outcome or small-and moderate-size, and for high-dimensional data where the number of predictors is usually higher than the number of sample observations.

To overcome the problem related to overfitting, some studies $[9,10]$ explored the use of penalized regression methods in risk prediction. Of them Ambler et al. [9] explored the use of two popular penalized regression methods, such as ridge [11] and lasso [12], in risk prediction for low-dimensional survival data with rare events and found that both methods improve calibration and discrimination compared with the ML based standard Cox models. Pavlou et al. [10] reviewed and evaluated ridge and lasso and their some extensions, such as elastic net, adaptive lasso etc [13-15], in risk prediction for low dimensional binary data with rare events and found that these methods can offers improvement, particularly for model overfitting, over the standard logistic regression model. Although these studies showed some improvement in risk prediction for rare-event data by using the penalized methods, there is no specific guidelines how risk prediction can be managed in the presence of separation, which frequently occur for such rare-event or sparse data. More specifically, the problem of separation, first reported by Albert and Anderson [16], is the case where one or more predictors have strong effects on response and hence (nearly ) perfectly predict the outcome of interest. Table 1 presents an example of both complete (perfect prediction) and quazi-complete separation (nearly perfect prediction) caused by a dichotomous predictor $X$ against binary outcome $Y$.

Separation may occur even if the data is large but there is sufficient number of strong predictors. The likelihood of separation is higher for categorical predictors with rare category compared to the continuous predictor [17]. When developing model in the presence of separation, $\mathrm{ML}$
Table 1 Example of separation due to a dichotomous predictor $X$ against outcome $Y$

\begin{tabular}{|c|c|c|c|c|c|c|c|}
\hline \multicolumn{4}{|c|}{ Complete separation } & \multicolumn{4}{|c|}{ Quazi-complete separation } \\
\hline & & \multicolumn{2}{|l|}{ Y } & \multicolumn{4}{|c|}{ Y } \\
\hline & & 1 & 0 & & & 1 & 0 \\
\hline \multirow{2}{*}{$x$} & A & 10 & 0 & \multirow{2}{*}{$x$} & A & 10 & 0 \\
\hline & $B$ & 0 & 10 & & $B$ & 2 & 8 \\
\hline
\end{tabular}

Number in each cell indicates number of observations

based logistic regression faces several problems [16, 18]. These includes lack of convergence of maximum likelihood and even if it converges it produces biased (sometimes infinite) estimate of the regression coefficient [17]. An alternative to the ML approach in this situation is Firth's penalized method [19]. This approach removes the first order term $\left(O\left(n^{-1}\right)\right)$ in the asymptotic bias expansion of the MLEs of the regression parameters by modifying the score equation with a penalty terms known as Jeffreys invariant prior. Heinze and Schemper [17] provided an application of Firth's method to the solution of the problem of separation in the logistic regression. Further the applications of Firth's method have been provided to proportional and conditional logistic regressions for situations with small-sample bias reduction and solution to problem of separation $[20,21]$.

However, one of the criticisms of Firth-type penalty in recent studies $[22,23]$ is that it depends on observed covariate data which can lead to artifacts such as estimates lying outside the range of prior median and the MLE (which is known as Bayesian non-collapsibility). An alternative to this, Greenland and Mansournia [22, 23] suggested $\log F(1,1)$ and $\log F(2,2)$ priors as default prior for logistic regression. As argued by the authors, the proposed $\log F$ priors are transparent, computationally simple, and reasonable for logistic regression. However, despite the attractive advantages of these penalized methods including Firth's method for sparse or small data sets, limited studies have been conducted to explore their use in risk prediction. This paper evaluates the predictive performance of these penalized methods for sparse data and compares the results with the ML based method and the other commonly used penalized method such as ridge. Although lasso is a commonly used method, it is popular for variable selection. Risk prediction and variable selection are different issues, and in this paper we have focused on prediction and hence excluded lasso.

This paper is organized as follows. The next section briefly describes all penalized methods under study. Then the following sections describe the simulation study, an illustration of the methodologies using stress ecocardiography data, and finally discussion and conclusions. 


\section{Methods}

\section{Maximum likelihood based logistic regression model}

Let $Y_{i},(i=1,2, \ldots, n)$, be a binary outcome $(0 / 1)$ for the $i$ th subject which follows Bernoulli distribution with the probability $\pi_{i}=\operatorname{Pr}\left(Y_{i}=1\right)$. The logistic regression model can be defined as

$$
\left.\operatorname{logit}\left[\pi_{i} \mid \boldsymbol{x}_{i}\right)\right]=\eta_{i}=\boldsymbol{\beta}^{T} \boldsymbol{x}_{i}
$$

where $\beta^{T}$ is a vector of regression coefficients of length $(k+1)$, and $\boldsymbol{x}_{i}$ is the $i$ th row vector of the predictor matrix $\boldsymbol{x}$ which has order $n \times(k+1)$. The term $\eta_{i}=\boldsymbol{\beta}^{T} \boldsymbol{x}_{i}$ is called as risk score or 'prognostic index'.

In standard MLE, the model is fitted by maximizing the $\log$ likelihood denoted by $l(\boldsymbol{\beta})$.

\section{Penalized methods for logistic regression model}

Whereas in penalized methods, $l(\boldsymbol{\beta})$ is maximized subject to constraints on the values of regression coefficients. The constraints are fixed in such a way so that the regression coefficient shrinks towards zero in comparison with MLE, which may help to alleviate overfitting. More specifically, the penalized regression coefficient is obtained by maximizing the penalized log likelihood denoted by $\nmid(\boldsymbol{\beta})-$ $\operatorname{pen}(\boldsymbol{\beta})$, where $\operatorname{pen}(\boldsymbol{\beta})$ is the 'penalty term'. The penalty term is the functional form of constraints. The penalized methods differ from each others in the choice of penalty term. The following subsection briefly discusses some popular penalized methods.

\section{Firth's penalized method}

In order to remove first order bias in MLEs of the regression coefficient, Firth [19] suggested to use penalty term $\frac{1}{2} \operatorname{trace}\left[I(\boldsymbol{\beta})^{-1} \partial I(\boldsymbol{\beta}) / \partial \beta_{j}\right]$ in the ML based score equation $U\left(\beta_{j}\right)=\partial l(\boldsymbol{\beta}) / \partial \beta_{j}=0$. The modified score equations are then $U\left(\beta_{j}\right)^{*}=U\left(\beta_{j}\right)+1 / 2 \operatorname{trace}\left[I(\boldsymbol{\beta})^{-1} \partial I(\boldsymbol{\beta}) / \partial \beta_{j}\right]=0$ $(j=1, \ldots, k)$, where $I(\beta)^{-1}$ is the inverse of information matrix evaluated at $\boldsymbol{\beta}$. The corresponding penalized log-likelihood function for the above modified score function is $l(\boldsymbol{\beta})+1 / 2 \log |I(\boldsymbol{\beta})|$. The penalty term used above is known as Jeffreys invariant prior and its influence is asymptotically negligible. The Firth type penalized MLE of $\boldsymbol{\beta}$ is thus $\hat{\boldsymbol{\beta}}=\operatorname{argmax}\{l(\boldsymbol{\beta})+1 / 2 \log |I(\boldsymbol{\beta})|\}$. This approach is known as bias preventive rather than corrective. However, Greenland and Mansournia [23] identified some problems in Jeffreys prior (equivalent to Firth's penalty term). These includes i)Jeffrey's prior is datadependent and includes correlation between covariates ii) the marginal prior for a given $\beta$ can change in opaque ways as model covariates are added or deleted, which may provide surprising results in sparse dataset, and iii) it is not clear how the penalty translate into prior probabilities for odds ratios.

\section{Penalized method based on $\log F$ prior}

To overcome these problems, Greenland and Mansournia [23] proposed a class of penalty functions $\operatorname{pen}(\boldsymbol{\beta})=$ $\ln \left(|I(\boldsymbol{\beta})|^{-m}\right)$ indexed by $m \geq 0$, which produce MLE for $m=0$. Then the penalized log-likelihood is equal to $l(\boldsymbol{\beta})+m \boldsymbol{\beta} / 2-m \ln \left(1+e^{\boldsymbol{\beta}}\right)$. They showed that the antilog of the penalty term $m \boldsymbol{\beta} / 2-m \ln \left(1+e^{\boldsymbol{\beta}}\right)$ is proportional to a $\log F(m, m)$ density for $\boldsymbol{\beta}$, which is the conjugate family for binomial logistic regression $[24,25]$. It is noted that the prior degrees of freedom $m$ in $\log F$ prior is exactly the number of observations added by the prior. Then the corresponding penalized ML estimate can be obtained as $\hat{\boldsymbol{\beta}}=\operatorname{argmax}\left\{l(\boldsymbol{\beta})+m \boldsymbol{\beta} / 2-m \ln \left(1+e^{\boldsymbol{\beta}}\right)\right\}$. This shows that $\hat{\boldsymbol{\beta}}$ has first order $\left(O\left(n^{-1}\right)\right)$ bias of zero for $m=1$, away from zero for $m<1$, and shrinks toward zero for $m>1$. This showed that $F(0,0)$ is equivalent to MLE, and $F(1,1)$ includes Jefrreys prior in one parameter model, for example, matched pair case-control. Greenland and Mansournia strongly argued against imposing a prior on the intercept to make sure that the mean predicted probability of binary condition is equal to the proportion of events. In this study, we focused on $F(1,1)$ and $F(2,2)$ prior for computational simplicity.

\section{Ridge penalized method}

Le Cessie and van Houwelingen [11] uses the penalty term as $\lambda_{2} \sum_{j=1}^{k} \beta_{j}^{2}$, where $\lambda_{2}$ is a tuning parameter that modulates the trade-off between the likelihood term and the penalty term and is usually selected as data-driven procedure such as cross validation. The ridge log-likelihood is thus defined as $l(\boldsymbol{\beta})-\lambda_{2} \sum_{j=1}^{k} \beta_{j}^{2}$ and hence $\hat{\boldsymbol{\beta}}=$ $\operatorname{argmax}\left\{l(\boldsymbol{\beta})-\lambda_{2} \sum_{j=1}^{k} \beta_{j}^{2}\right\}$. Ridge was initially developed to solve the problems due to multicolinearity. However, it shrinks the regression coefficient towards nearly zero and hence can be performed well to alleviate overfitting in risk prediction in the scenario with correlated predictors.

\section{Evaluating predictive performance}

Three common approaches to evaluate the predictive performance of a risk model [26]. These are i) calibration (the agreement between the observed and predicted risk in a group of subjects) ii) discrimination (the ability of model to distinguish between low-and high-risk patients) iii) overall prediction accuracy.

Calibration: We assessed calibration by calculating calibration slope, which can be obtained by re-fitting a binary logistic regression model with linear predictor or prognostic index (PI) derived from the original model as the only predictor. The estimated slope $\hat{\beta}_{P I}$ is the calibration slope. If $\hat{\beta}_{P I}=1$, it suggests perfect calibration; $\hat{\beta}_{P I}<1$ suggests overfitting, and $\hat{\beta}_{P I}>1$ suggest underfitting. 
Discrimination: We assessed discriminating ability of the model by quantifying the area under receiver operating characteristic curve (AUC), graph of sensitivity (true-positive rate) versus one minus specificity (truenegative rate) evaluated at consecutive threshold values of the predicted risk score or probability derived from the model. Alternatively AUC can be obtained by quantifying the probability that, for a randomly selected pair of subjects, the subject who experienced the event of interest had higher predicted risk derived from the model than those without experiencing the event. A value $\mathrm{AUC}=0.5$ indicates no discrimination and 1 suggest perfect discrimination.

Overall predictive performance: The overall prediction accuracy is quantified using Brier score, which is the mean of the squared difference between the observed and predicted risk for each patient derived from the model. The lower the BS, the better the prediction of a model and $\mathrm{BS}=0$ indicates perfect prediction. For ease of interpretation we reported root $\mathrm{BS}(\mathrm{rBS})$. In addition to the $\mathrm{rBS}$, we also reported average predictive probability (APP) of the model to see how the predicted value differ from the corresponding observed value.

\section{Software}

All the analyses and simulations were conducted in Stata version 12. Several Stata packages and functions were used to fit the models in different methods under study. These includes 'logit', 'firthlogit', 'penlogit', and 'plogit' along with 'plsearch' for MLE, FIRTH, $\log F$ and RIDGE, respectively. The calculation of calibration slope and Brier score were performed using self written Stata code and AUC using the package 'roctab'.

\section{Results}

\section{Example data: stress echocardiography data}

The dataset used for simulation and illustration is in public domain and originally extracted from the study conducted by Krivokapich et al. [27] where the aim was to quantify the prognostic value of dobutamine stress echocardiography (DSE) in predicting cardiac events in 558 patients (male 220 and female 338) with known or suspected coronary artery disease. The responses of interest whether or not a patient suffered from either of 'death due to cardiac arrest', or ' myocardial infarction (MI), or ' revascularization by percutaneous transluminal coronary angioplasty (PTCA)' or 'coronary artery bypass grafting surgery (CABG)' over the next year after having the test. There were 24 patients with cardiac death, 28 with MI, 27 with PTCA, 33 with CABG and 89 with any cardiac event (Cevent), which implies that the each of the events was rare. The main predictor of interest are age, history of hypertension (HT: yes/no) and diabetics mellitus (DM: yes/no), history of prior MI (yes/no) and PTCA (yes/no), status of DSE test (positive DSE:positive/negative), wall motion anamoly on echocardiogram (rest WMA:yes/no), ejection fraction on dobutamine(Dobutamine EF), and base ejection fraction (base EF).

\section{Simulation study}

The performance of the penalized methods in risk prediction over standard ML based logistic regression were investigated using a simulation study. We conducted simulation i) firstly to assess and compare the properties of the regression coefficients of the different methods (MLE, FIRTH, $\log F(1,1), \log F(2,2)$, RIDGE) under study and ii) secondly to assess and compare the predictive performance between the methods.

\section{Assessing the properties of the regression coefficients}

To assess the properties of the regression coefficients such as bias and mean squared error (MSE), we generated two independent predictors of which one is continuous $\left(X_{1}\right)$ generated from standard normal and the other is dichotomous $\left(X_{2}\right)$ generated from Bernoulli distribution with $50 \%$ events. We then generated binary response from Bernoulli distribution with probability $\pi_{i}(i=1, \ldots, n)$ calculated from true logistic model $\operatorname{logit}(\pi)=\beta_{0}+\beta_{1} X_{1}+\beta_{2} X_{2}$, where $\beta_{1}=0.30$ and $\beta_{2}=0.9$. With this combination, the binary covariate created separation for some of the simulated datasets particularly with low prevalence. The value of $\beta_{0}$ vary to generate data with varying level of prevalence. The scenarios were created by varying the prevalence, on an average, $(p)$ as 5.5, 11.520 .4 and $39.6 \%$ for a fixed sample size $n=120$. For each scenario, 1000 datasets were generated and all regression approaches under study were fitted to each dataset. When fitting RIDGE the respective tuning parameters were selected through 10 -fold cross validation. The estimates of the regression coefficients of the respective models were obtained as the mean over the number of simulations where convergence achieved. Noted that only MLE and RIDGE were failed to converge (due to low prevalence or separation or both) in some datasets, and the maximum failure rate for MLE and RIDGE were 13 and 51\% , respectively for the lowest prevalence scenario. The failure rate decreases as the prevalence increases. Finally the relative bias (\%) and mean squared error (MSE) of the estimates were reported and compared if the performance vary across the scenarios.

The results in Table 2 showed that the RIDGE estimator, in general, provides the highest amount of relative bias (\%), which is followed by the MLE and $\log F(2,2)$ whereas FIRTH and $\log F(2,2)$ provides negligible bias. For the coefficient of the dichotomous predictor $\left(\beta_{2}\right) \log F(2,2)$ provides more bias compared to those for continuous predictor $\left(\beta_{1}\right)$. The amount of bias, in general, is the highest 


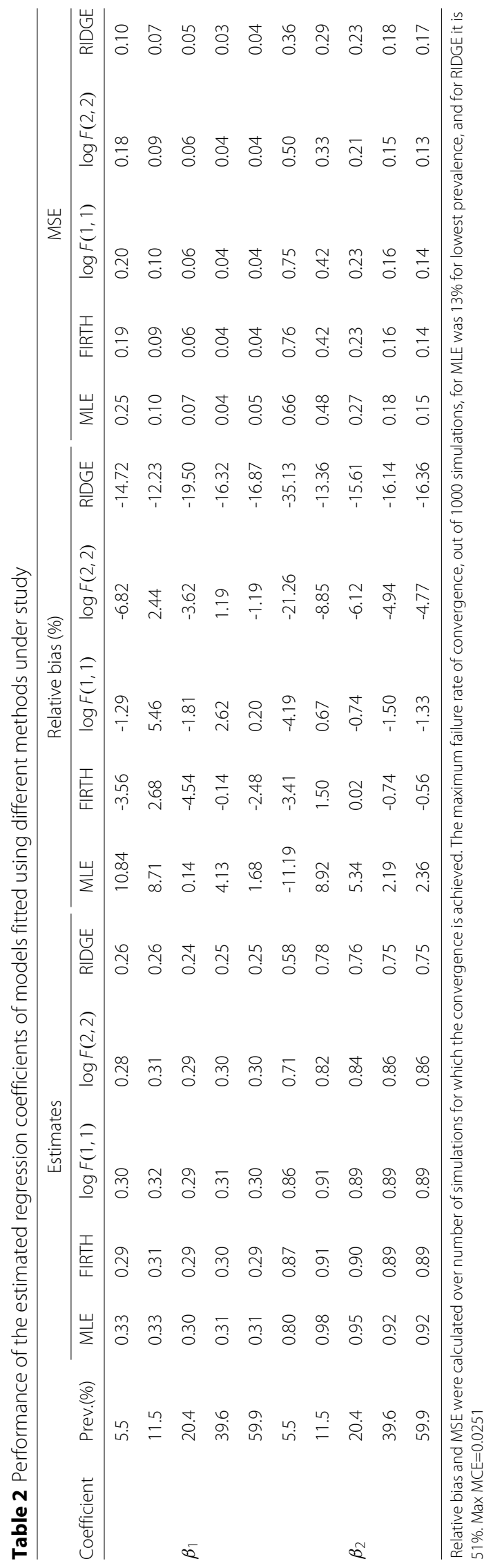


for the low prevalence data and the lowest for the high prevalence data. However, the RIDGE, in general, produces the lowest MSE, and the highest MSE is produced by the MLE for $\beta_{1}$ and by FIRTH for $\beta_{2}$. The amount of MSE, in general, decreases with the increasing prevalence.

\section{Assessing the predictive performance}

To assess the predictive performance of the methods, we conducted two simulation series following the simulation design in Pavlou et al. [10] used for similar type of study. The first simulation series is based on the real stress echocardiography data where only responses were generated and in the second simulation series we generated both covariates and responses.

\section{Stress echocardiography simulation}

In the first simulation series based on real data, we simulated data and evaluated the predictive performance of the models for different EPV scenarios using the following steps:

(i) Fit the following logistic regression model for the response "any cardiac event" with Firth's penalized method (to avoid bias in the estimate of the regression coefficient) to obtain the true model:

$\operatorname{logit}(\operatorname{Pr}($ Cevent $=1))=\beta_{0}+\beta_{1}$ dobef $+\beta_{2} w m a+\beta_{3}$ posse

$$
+\beta_{4} b s e f+\beta_{5} h t+\beta_{6} \text { age }
$$

(ii) To create a training data, choose the EPV and prevalence (prev), and then calculate sample size for the respective EPV given the number of predictors $p$ as $n=\frac{E P V \times p}{\text { prev }}$. Sample with replacement the $n$ values of the covariates in the true model from original data. For each of the $n$ values of the covariates, simulate new responses from Bernoulli distribution with the probability calculated from the fitted model.

However, replace the value of $\beta_{0}$ by -0.65 to confirm the prevalence of the response (prev), on an average, $15.5 \%$ for all EPV scenarios.

(iii) With this combination, check and record if separation occurred due to any of the binary covariates ('posse' or 'wma', or 'ht' or combination of them). Otherwise to create separation, enlarge the true value of the respective coefficient of the binary covariate to some extents. Note that the chances of separation is expected to increase with decreasing EPV value.

(iv) To create a test dataset, sample with replacement $m \times n$ ( $m$ times of the original data of size $n=558$, we considered $m=2$ ) values of the covariates. Then simulate the corresponding new responses from the same true model used for simulating training data.

(v) Repeat the steps (ii)-(iii) to produce 1000 training and 1000 test datasets.

(vi) Fit the risk models ( using MLE and all types of penalized regression methods under study) to each of the training data sets and check whether convergence was achieved. Then evaluate their predictive performance (if convergence achieved) by means of calibration slope, AUC, root Brier score, and average predictive probability (APP) using the corresponding test dataset. Summarize the predictive performance over the number of simulations for which convergence is achieved.

The predictive performance of all regression methods was investigated against $E P V=2,3,5,10$ to see if the performance vary across the scenarios. When the predictive performance against EPV was assessed by means of calibration slope, the MLE showed poor performance by producing overfitted model (calibration slope substantially lower than 1) for EPV=2, 3, 5 (Fig. 1). All penalized methods offered improvement to some extents except the RIDGE which produced underfitted model ( the average value of the calibration slope greater than 1 with high SD). In addition, the RIDGE failed to converge for the maximum $8.4 \%$ of the simulations particularly when $E P V=2$. Almost equal improvement was offered by the Firth-type and both the $\log F(1,1)$ and $\log F(2,2)$ penalized methods. In general all methods including MLE showed almost equal performance in terms of calibration for high EPV $(E P V=10)$. When the predictive performance (discriminatory ability) was assessed through AUC, all penalized methods showed better performance with greater AUC than MLE for the low EPV scenarios (Fig. 2). Of them the RIDGE provided highest AUC value. However, the amount of improvement in the discrimination, in general, was comparatively lower than that for calibration. All methods perform almost equally for high EPV $(\mathrm{EPV}=10)$. Similarly the penalized methods offered improvement in the overall predictive performance for individual prediction assessed through rBS to some extents for low EPV (Fig. 3). Of them, the RIDGE offered greater improvement. However, for low EPV while both the $\log F(1,1)$ and $\log F(2,2)$ penalized methods provided accurate estimate of the true average predicted probability (APP) 15.2\%, the FIRTH-type penalized method overestimate the true value. The amount of bias in FIRTH-type estimate is even larger than that produced by MLE and RIDGE (Fig. 4).

\section{Further simulation}

In the second simulation series with the same EPV scenarios, we simulated both covariates and response under two predictive models, one with weak predictive ability and the other with strong predictive ability, using the following steps:

(i) For creating training data, choose the EPV and prevalence (prev) and calculate sample size ( $n$ ) for 

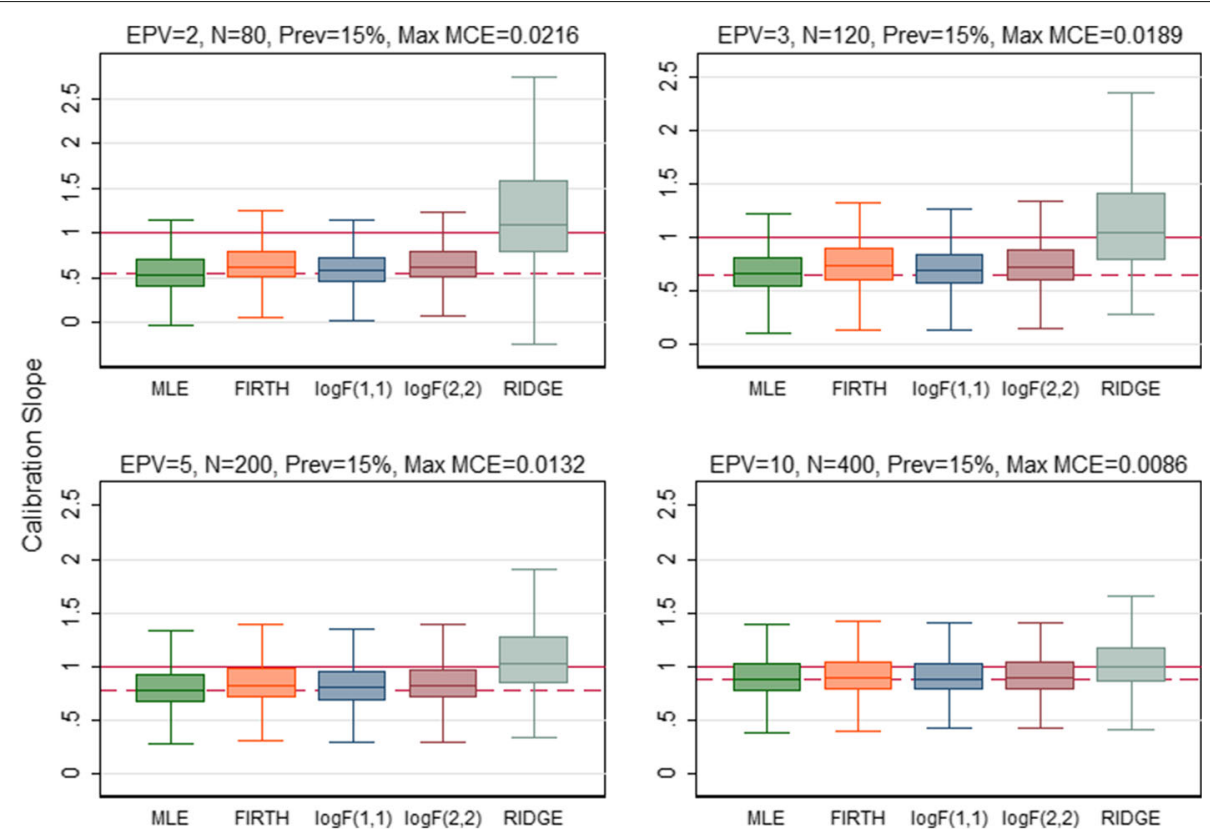

Fig. 1 Performance of the methods was assessed using calibration slope and compared. Results were summarized over the number of simulations for which convergence is achieved. The maximum failure rate of convergence for RIDGE, out of 1000 simulations, is $8.4 \%$ when EPV=2. The values outside the whisker were not plotted to make the plot readable. The horizontal dash line is the median calibration slope for MLE and the solide line is the optimal value

the given EPV value and the number of predictors using the same formula previously used.

(ii) For each observation in training data, first simulate three continuous predictors $\left(X_{1}, X_{2}, X_{3}\right)$ independently from standard normal distribution and two binary predictors $\left(X_{4}, X_{5}\right)$ independently from Bernoulli distribution one with low (20\%) and the other with high (60\%) prevalence.

(iii) Simulate the corresponding responses from Bernoulli distribution with probability calculated from the true model:

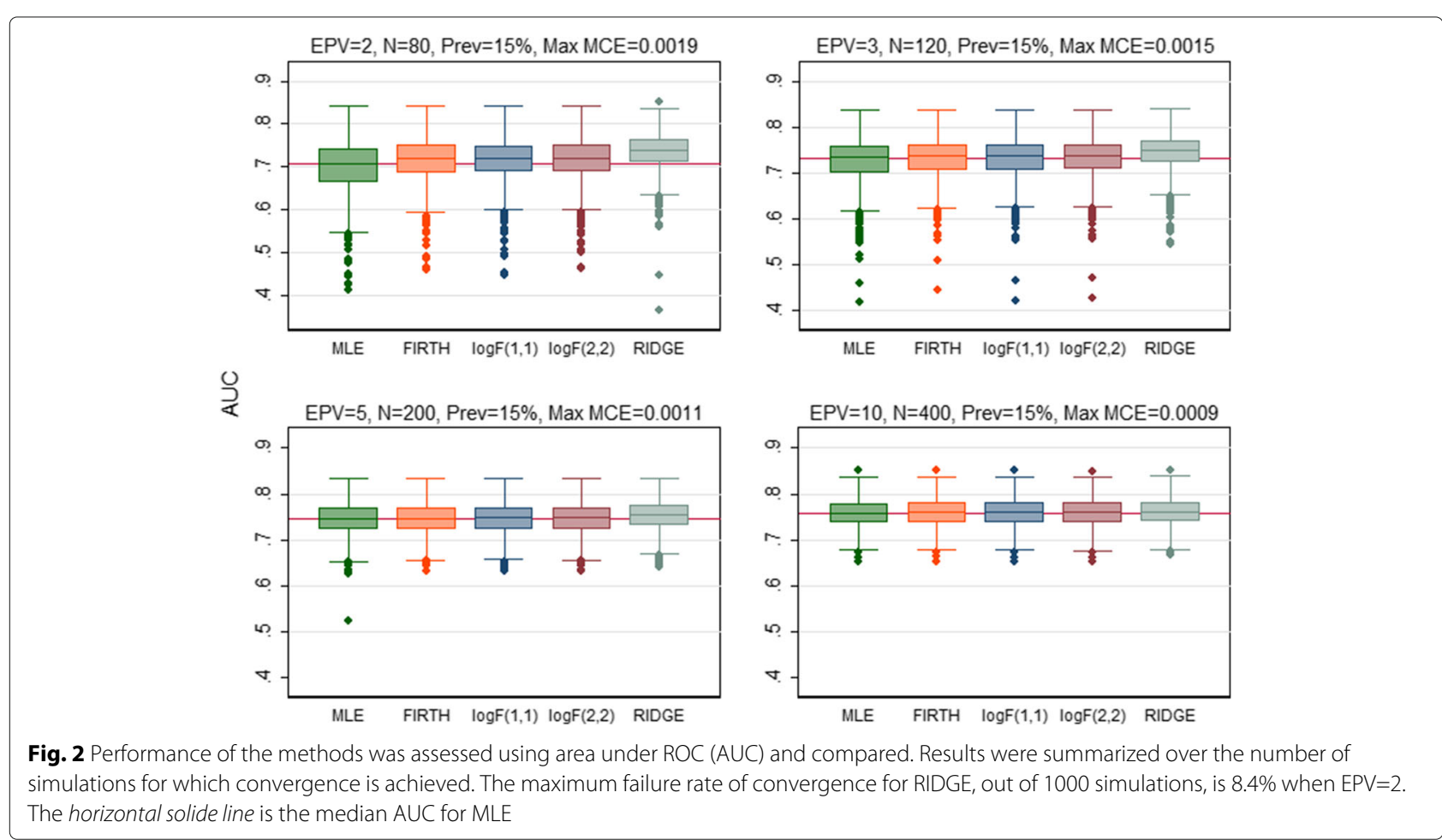



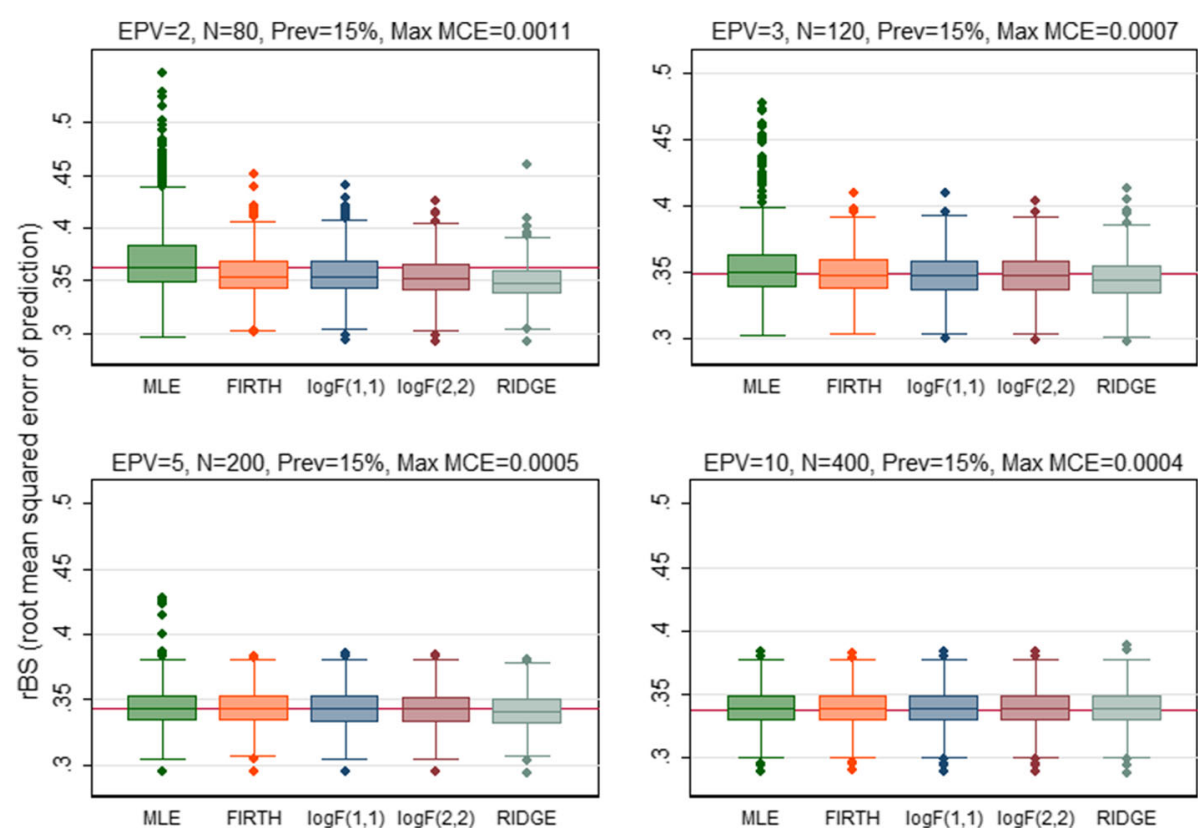

Fig. 3 Performance of the methods was assessed using root Brier score (rBS) and compared. Results were summarized over the number of simulations for which convergence is achieved. The maximum failure rate of convergence for RIDGE, out of 1000 simulations, is $8.4 \%$ when $E P V=2$. The horizontal solide line is the median rBS for MLE

$\operatorname{logit}(\pi)=\beta_{0}+\beta_{1} X_{1}+\beta_{2} X_{2}+\beta_{3} X_{3}+\beta_{4} X_{4}+\beta_{5} X_{5}$.

For the model with weak predictive ability, the values of the regression coefficient were set as $\beta_{0}=-1.5 \beta_{1}=$ $0.2, \beta_{2}=0.5 \beta_{3}=-0.03, \beta_{4}=0.05$ and $\beta_{5}=-0.6$ and for the model with strong predictive ability, the corresponding true values were set as $\beta_{0}=-3.5$, $\beta_{1}=1.2, \beta_{2}=-0.9 \beta_{3}=0.9, \beta_{4}=1.2$ and $\beta_{5}=1.2$. In each case, the value of the $\beta_{0}$ confirms the desired prevalence of the response. With this combination,
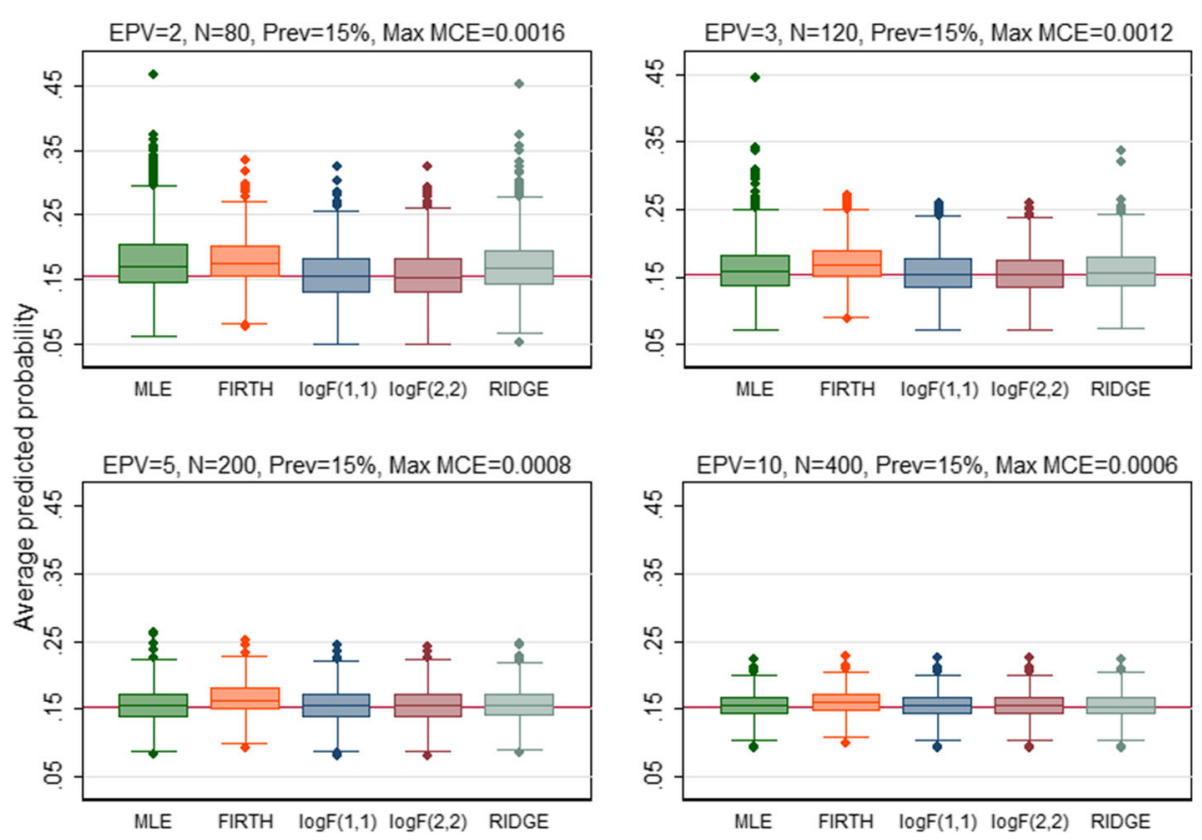

Fig. 4 Performance of the methods was assessed using Average Predicted Probability (APP) and compared. Results were summarized over the number of simulations for which convergence is achieved. The maximum failure rate of convergence for RIDGE, out of 1000 simulations, is $8.4 \%$ when $E P V=2$. The horizontal solide line is the observed APP 
the binary covarites $X_{5}$ in the model with weak predictive ability and $X_{4}$ in the model with strong predictive ability create separation in some of the simulations. Check and record if separation occurred.

(iv) Create test data with size 1000 (much larger than the training data) for the similar level of EPV and prevalence. For each observation in the test data, simulate the same predictors as in the test data and the corresponding response from the same true model.

(v) Repeat the steps (ii)-(iv) to produce 1000 training and 1000 test datasets

(vi) Fit risk models (using all methods) using training data, count if convergence was achieved for the respective model, and evaluate their predictive performance (if convergence was achieved in training data) using test data as before. Finally summarize the predictive performance over the number of simulations for which convergence is achieved.

The results revealed that, for both predictive models (weak and strong predictive abilities), all the penalized methods offered improvement in calibration over MLE for low EPV, except for the RIDGE which in turn provided underfitted model (calibration slope grater than 1 with high SD) (Table 3). The amount of improvement by the other penalized methods was almost equal. However, all the penalized methods except the RIDGE offered negligible improvement in the discrimination for low EPV. Similarly all the penalized methods showed improvement to some extents in the overall predictive performance by lowering the rBS value compared to that for MLE. For both predictive model, the average predicted probability (APP) estimated by the both the $\log F(1,1)$ and $\log F(2,2)$ were almost equal to the average observed probability, however the Firth-type penalized method introduced positive bias in the estimate of the average probability. The amount of bias was even larger than that for MLE and RIDGE. In case of both models, the maximum failure of convergence (due to separation or low EPV or both) was reported for RIDGE.

\section{Illustration using stress echocardiography data}

The aim is to derive risk models using different penalized methods discussed earlier and the standard MLE to predict the risk of having a cardiac event and then to evaluate and compare their predictive performance. We fitted separate models for predicting the risk of each of the four cardiac events and a model for the risk of any of the events using each regression approaches; that is, a total of five models for each of the binary events were fitted using six different regression methods under study and altogether 25 models for all five binary responses.

The models were fitted using training data (contains
$60 \%$ of total data randomly selected) and their predictive performance were evaluated using test data (contains rest of 40\%). The associated predictors for each cardiac event were selected based on the information from literature and results of likelihood ratio test (LRT). Different combinations of predictors were tested using LRT to come up with a final model for each cardiac event. Then the same model was then fitted in training data using six different methods. Note that quasi-complete separation due to binary predictors in training data was identified for the responses 'PTCA' and ' cardiac death', and hence, in case of convergence failure for RIDGE or MLE, the estimates reported are based on the last iteration. The estimated coefficients of the respective model are then summarized in Table 4. For all types of response, the estimated regression coefficients for MLE is larger than all penalized methods. Because all the methods shrink the coefficient towards zero. The amount of shrinking was higher for the RIDGE in the most of the cases. However, the main purpose here is to evaluate the predictive performance of the methods rather than comparing their estimated regression coefficients. The predictive performance of all models were then evaluated using test data, and the results were summarized in Table 5.

It is observed from results in Table 5 that all models faced the problem of overfitting (calibration slope $<<$ 1) particularly for those response for which the EPV is low $(\mathrm{EPV}<10)$. The amount of overfitting is lower for all penalized methods compared to MLE. In terms of discrimination all methods including MLE provided comparable results. For all types of response, the greater improvement was observed in the calibration (calibration slope) compared to those in both discrimination (AUC) and overall performance (BS). Firth methods produced higher value of the average predicted probability (APP) for all type of responses.

The probable reason for producing overfitted models (very low value of the calibration slope) even for the penalized methods is that the size of the test data and particularly the number of events for all types of response were very small compared to the number of events (approximately 100) required for correct estimation of the predictive accuracy measures [28]. Therefore, further the predictive performance of all models were evaluated in test data consisting of larger sample size and number of events compared to the previous test data. This was created by expanding 5 times the original (previous) test data so that the required number of events is achieved. In this procedure each subject replaced his/her information for the other 4 subjects. The results showed that calibration slope was comparatively more closer to 1 (suggesting improvement in calibration) for the penalized methods for all types of responses, particularly for which EPV was 
Table 3 Performance measures for the model s with both weak and strong predictive ability. Results were summarized over the number of simulations for which convergence is achieved. The maximum failure rate of convergence for RIDGE with weak predictive ability, out of 1000 simulations, is $40 \%$ for the lowest EPV

\begin{tabular}{|c|c|c|c|c|c|c|c|c|c|c|c|}
\hline \multicolumn{12}{|c|}{ Model with weak predictive ability } \\
\hline & & \multicolumn{5}{|c|}{ Calibration slope, Max MCE $=0.0235$} & \multicolumn{5}{|c|}{ AUC, Max MCE $=0.0012$} \\
\hline \multicolumn{2}{|l|}{ EPV $(N)$} & MLE & FIRTH & $\log F(1,1)$ & $\log F(2,2)$ & RIDGE & MLE & FIRTH & $\log F(1,1)$ & $\log F(2,2)$ & RIDGE \\
\hline \multirow[t]{2}{*}{$2(67)$} & Mean & 0.367 & 0.414 & 0.383 & 0.424 & 1.029 & 0.606 & 0.605 & 0.605 & 0.607 & 0.628 \\
\hline & SD & 0.277 & 0.303 & 0.281 & 0.302 & 0.847 & 0.060 & 0.058 & 0.059 & 0.059 & 0.042 \\
\hline \multirow[t]{2}{*}{$3(100)$} & Mean & 0.472 & 0.512 & 0.487 & 0.517 & 1.027 & 0.613 & 0.613 & 0.613 & 0.614 & 0.626 \\
\hline & SD & 0.305 & 0.326 & 0.311 & 0.324 & 0.757 & 0.054 & 0.054 & 0.054 & 0.054 & 0.041 \\
\hline \multirow[t]{2}{*}{$5(167)$} & Mean & 0.621 & 0.658 & 0.637 & 0.658 & 1.055 & 0.629 & 0.630 & 0.630 & 0.630 & 0.635 \\
\hline & SD & 0.317 & 0.328 & 0.317 & 0.323 & 0.667 & 0.046 & 0.046 & 0.046 & 0.046 & 0.039 \\
\hline \multirow[t]{3}{*}{ 10(334) } & Mean & 0.797 & 0.814 & 0.801 & 0.812 & 1.076 & 0.645 & 0.645 & 0.645 & 0.646 & 0.646 \\
\hline & SD & 0.286 & 0.289 & 0.282 & 0.286 & 0.504 & 0.037 & 0.037 & 0.037 & 0.037 & 0.035 \\
\hline & & \multicolumn{5}{|c|}{ root Brier Score, Max MCE =0.0007 } & \multicolumn{5}{|c|}{ APP (True 0.152), Max MCE =0.0015 } \\
\hline$E P V(N)$ & & MLE & FIRTH & $\log F(1,1)$ & $\log F(2,2)$ & RIDGE & MLE & FIRTH & $\log F(1,1)$ & $\log F(2,2)$ & RIDGE \\
\hline \multirow[t]{2}{*}{$2(67)$} & Mean & 0.370 & 0.369 & 0.367 & 0.365 & 0.360 & 0.159 & 0.178 & 0.154 & 0.153 & 0.156 \\
\hline & SD & 0.022 & 0.019 & 0.019 & 0.018 & 0.017 & 0.045 & 0.041 & 0.044 & 0.044 & 0.044 \\
\hline \multirow[t]{2}{*}{$3(100)$} & Mean & 0.363 & 0.362 & 0.361 & 0.360 & 0.358 & 0.156 & 0.171 & 0.154 & 0.154 & 0.155 \\
\hline & SD & 0.018 & 0.017 & 0.017 & 0.017 & 0.016 & 0.035 & 0.033 & 0.035 & 0.035 & 0.035 \\
\hline \multirow[t]{2}{*}{$5(167)$} & Mean & 0.357 & 0.357 & 0.357 & 0.356 & 0.355 & 0.153 & 0.163 & 0.153 & 0.153 & 0.152 \\
\hline & SD & 0.017 & 0.016 & 0.017 & 0.016 & 0.016 & 0.028 & 0.027 & 0.027 & 0.027 & 0.027 \\
\hline \multirow[t]{2}{*}{$10(334)$} & Mean & 0.354 & 0.354 & 0.354 & 0.354 & 0.354 & 0.151 & 0.157 & 0.151 & 0.151 & 0.151 \\
\hline & SD & 0.016 & 0.015 & 0.016 & 0.016 & 0.015 & 0.020 & 0.019 & 0.020 & 0.020 & 0.019 \\
\hline \multicolumn{12}{|c|}{ Model with strong predictive ability } \\
\hline & & \multicolumn{5}{|c|}{ Calibration slope, Max MCE $=0.0344$} & \multicolumn{5}{|c|}{ AUC, Max MCE $=0.0024$} \\
\hline EPV $(N)$ & & MLE & FIRTH & $\log F(1,1)$ & $\log F(2,2)$ & RIDGE & MLE & FIRTH & $\log F(1,1)$ & $\log F(2,2)$ & RIDGE \\
\hline \multirow[t]{2}{*}{$2(67)$} & Mean & 0.659 & 0.825 & 0.784 & 0.890 & 1.252 & 0.831 & 0.831 & 0.832 & 0.834 & 0.832 \\
\hline & SD & 0.296 & 0.310 & 0.268 & 0.273 & 0.742 & 0.039 & 0.039 & 0.038 & 0.037 & 0.037 \\
\hline \multirow[t]{2}{*}{$3(100)$} & Mean & 0.774 & 0.888 & 0.857 & 0.931 & 1.125 & 0.845 & 0.845 & 0.846 & 0.846 & 0.845 \\
\hline & SD & 0.236 & 0.251 & 0.231 & 0.233 & 0.292 & 0.028 & 0.028 & 0.028 & 0.028 & 0.028 \\
\hline \multirow[t]{2}{*}{$5(167)$} & Mean & 0.868 & 0.934 & 0.917 & 0.963 & 1.066 & 0.854 & 0.854 & 0.854 & 0.855 & 0.854 \\
\hline & SD & 0.218 & 0.226 & 0.216 & 0.217 & 0.224 & 0.024 & 0.023 & 0.023 & 0.023 & 0.023 \\
\hline \multirow[t]{3}{*}{ 10(334) } & Mean & 0.933 & 0.959 & 0.955 & 0.979 & 1.016 & 0.860 & 0.860 & 0.860 & 0.860 & 0.860 \\
\hline & SD & 0.167 & 0.169 & 0.166 & 0.167 & 0.159 & 0.022 & 0.022 & 0.022 & 0.022 & 0.022 \\
\hline & & \multicolumn{5}{|c|}{ root Brier Score, Max MCE=0.0009 } & & APP (T & 0.162), Max & $E=0.0014$ & \\
\hline EPV $(N)$ & & MLE & FIRTH & $\log F(1,1)$ & $\log F(2,2)$ & RIDGE & MLE & FIRTH & $\log F(1,1)$ & $\log F(2,2)$ & RIDGE \\
\hline $2(67)$ & Mean & 0.338 & 0.331 & 0.330 & 0.327 & 0.328 & 0.172 & 0.182 & 0.164 & 0.163 & 0.167 \\
\hline & SD & 0.030 & 0.022 & 0.021 & 0.020 & 0.019 & 0.045 & 0.040 & 0.042 & 0.042 & 0.042 \\
\hline $3(100)$ & Mean & 0.323 & 0.321 & 0.321 & 0.320 & 0.320 & 0.165 & 0.175 & 0.163 & 0.163 & 0.164 \\
\hline & SD & 0.018 & 0.016 & 0.017 & 0.016 & 0.016 & 0.033 & 0.032 & 0.033 & 0.033 & 0.032 \\
\hline $5(167)$ & Mean & 0.316 & 0.315 & 0.315 & 0.315 & 0.315 & 0.163 & 0.170 & 0.163 & 0.163 & 0.163 \\
\hline & SD & 0.016 & 0.015 & 0.015 & 0.015 & 0.015 & 0.026 & 0.025 & 0.025 & 0.025 & 0.025 \\
\hline 10(334) & Mean & 0.310 & 0.310 & 0.310 & 0.310 & 0.310 & 0.163 & 0.166 & 0.163 & 0.163 & 0.163 \\
\hline & SD & 0.016 & 0.015 & 0.015 & 0.015 & 0.015 & 0.019 & 0.019 & 0.019 & 0.019 & 0.019 \\
\hline
\end{tabular}


Table 4 Modeling the risk of cardiac events. Estimate of the regression coefficients with SE in the parenthesis

\begin{tabular}{|c|c|c|c|c|c|}
\hline & \multicolumn{5}{|c|}{ Modeling the risk of $\mathrm{Ml}$} \\
\hline & MLE & FIRTH & $\log F(1,1)$ & $\log F(2,2)$ & RIDGE \\
\hline \multirow[t]{2}{*}{ Dobutamine EF } & -0.0503 & -0.0492 & -0.0508 & -0.0513 & -0.0413 \\
\hline & $(0.0183)$ & $(0.0178)$ & $(0.0183)$ & $(0.0182)$ & $(0.0161)$ \\
\hline \multirow[t]{2}{*}{ Positive DSE } & 1.272 & 1.241 & 1.185 & 1.109 & 0.994 \\
\hline & $(0.549)$ & $(0.531)$ & $(0.533)$ & $(0.518)$ & $(0.469)$ \\
\hline \multirow[t]{2}{*}{ Hist. of $\mathrm{HT}$} & 1.115 & 0.923 & 0.973 & 0.866 & 0.657 \\
\hline & $(0.789)$ & $(0.716)$ & $(0.716)$ & $(0.662)$ & $(0.542)$ \\
\hline \multirow[t]{3}{*}{ Intercept } & -1.253 & -1.028 & -1.057 & -0.901 & -1.243 \\
\hline & $(1.351)$ & $(1.284)$ & $(1.307)$ & $(1.275)$ & $(1.132)$ \\
\hline & \multicolumn{5}{|c|}{ Modeling the risk of $C A B G$} \\
\hline \multirow[t]{2}{*}{ Dobutamine EF } & -0.0634 & -0.0506 & -0.0518 & -0.0523 & -0.0420 \\
\hline & $(0.0181)$ & $(0.0177)$ & $(0.0181)$ & $(0.0181)$ & $(0.0161)$ \\
\hline \multirow[t]{2}{*}{ Positive DSE } & 1.568 & 1.190 & 1.137 & 1.068 & 0.971 \\
\hline & $(0.551)$ & $(0.529)$ & $(0.531)$ & $(0.516)$ & $(0.468)$ \\
\hline \multirow[t]{3}{*}{ Intercept } & 0.272 & -0.224 & -0.206 & -0.145 & -0.683 \\
\hline & $(1.122)$ & $(1.120)$ & $(1.140)$ & $(1.131)$ & $(1.029)$ \\
\hline & \multicolumn{5}{|c|}{ Modeling the risk of PTCA } \\
\hline \multirow[t]{2}{*}{ Positive DSE } & 0.825 & 0.820 & 0.770 & 0.722 & 0.579 \\
\hline & $(0.498)$ & $(0.481)$ & $(0.483)$ & $(0.470)$ & $(0.409)$ \\
\hline \multirow[t]{2}{*}{ Base EF } & -0.0381 & -0.0375 & -0.0389 & -0.0396 & -0.0306 \\
\hline & $(0.0204)$ & $(0.0198)$ & $(0.0202)$ & $(0.0201)$ & $(0.0168)$ \\
\hline \multirow[t]{2}{*}{ Hist. of Ml } & 1.168 & 1.125 & 1.118 & 1.072 & 0.867 \\
\hline & $(0.533)$ & $(0.517)$ & $(0.515)$ & $(0.499)$ & $(0.412)$ \\
\hline \multirow[t]{2}{*}{ Hist of PTCA } & 1.304 & 1.310 & 1.211 & 1.127 & 1.087 \\
\hline & $(0.617)$ & $(0.591)$ & $(0.602)$ & $(0.588)$ & $(0.555)$ \\
\hline \multirow[t]{3}{*}{ Intercept } & -1.754 & -1.661 & -1.646 & -1.548 & -1.809 \\
\hline & $(1.184)$ & $(1.151)$ & $(1.167)$ & $(1.153)$ & $(0.968)$ \\
\hline & \multicolumn{5}{|c|}{ Modeling the risk of cardiac death } \\
\hline \multirow[t]{2}{*}{ Positive DSE } & 1.084 & 1.061 & 1.026 & 0.974 & 0.873 \\
\hline & $(0.489)$ & $(0.474)$ & $(0.478)$ & $(0.467)$ & $(0.436)$ \\
\hline \multirow[t]{2}{*}{ Hist. of DM } & 1.083 & 1.047 & 1.025 & 0.973 & 0.784 \\
\hline & $(0.495)$ & $(0.480)$ & $(0.481)$ & $(0.468)$ & $(0.419)$ \\
\hline \multirow[t]{2}{*}{ Age } & 0.0347 & 0.0328 & 0.0344 & 0.0342 & 0.0229 \\
\hline & $(0.0240)$ & $(0.0236)$ & $(0.0238)$ & $(0.0236)$ & $(0.0188)$ \\
\hline \multirow[t]{2}{*}{ Intercept } & -6.040 & -5.783 & -5.965 & -5.899 & -4.960 \\
\hline & $(1.787)$ & $(1.750)$ & $(1.769)$ & $(1.753)$ & (1.379) \\
\hline \multirow[t]{2}{*}{ Positive DSE } & 1.064 & 1.045 & 1.047 & 0.989 & 0.940 \\
\hline & $(0.332)$ & $(0.327)$ & $(0.327)$ & $(0.264)$ & $(0.248)$ \\
\hline \multirow[t]{2}{*}{ Dobutamine EF } & -0.0381 & -0.0372 & -0.0384 & -0.0364 & -0.0333 \\
\hline & $(0.0131)$ & $(0.0128)$ & $(0.0130)$ & $(0.0103)$ & $(0.00941)$ \\
\hline
\end{tabular}


Table 4 Modeling the risk of cardiac events. Estimate of the regression coefficients with SE in the parenthesis (Continued)

\begin{tabular}{|c|c|c|c|c|c|}
\hline \multirow[b]{2}{*}{ Rest WMA } & \multicolumn{5}{|c|}{ Modeling the risk of any cardiac event } \\
\hline & -0.779 & -0.758 & -0.755 & -0.839 & -0.762 \\
\hline & $(0.429)$ & $(0.421)$ & $(0.419)$ & $(0.336)$ & $(0.293)$ \\
\hline \multirow[t]{2}{*}{ Hist. of HT } & 0.823 & 0.781 & 0.794 & 0.728 & 0.632 \\
\hline & $(0.393)$ & $(0.384)$ & $(0.384)$ & $(0.302)$ & $(0.276)$ \\
\hline \multirow[t]{2}{*}{ Intercept } & 0.107 & 0.115 & 0.151 & 0.0102 & -0.0980 \\
\hline & $(0.833)$ & $(0.818)$ & $(0.829)$ & $(0.655)$ & $(0.611)$ \\
\hline
\end{tabular}

high (results not showed). Similar results were obtained for the AUC and Brier score for all types of models of all responses.

\section{Discussion}

Penalized regression methods (such as RIDGE and LASSO) has increasingly being used for developing models for high dimensional data where the number of predictors is higher than the number of subjects. Furthermore several studies $[29,30]$ have also been conducted to make relative comparison between the methods for high dimensional case and found that RIDGE performed well when data have highly correlated predictors and LASSO performed well when variable selection is required. Although few studies $[9,10]$ evaluated RIDGE, LASSO and others in risk prediction for low-dimensional survival and binary data with few events, however, they often ignored Firth-and $\log F$-type (such as $\log F(1,1)$ and $\log F(2,2)$ ) penalized methods, despite their attractive advantages in reducing finite sample bias in the estimated regression coefficient and solving problem of separation that commonly occurs in low-dimensional small or sparse datasets. This paper explored the use of these methods in risk prediction for small and sparse data and compared their predictive performance with MLE and the other penalized method (RIDGE). In particular we focused on comparing the predictive performance of the methods through assessing calibration, discrimination and overall predictive performance when EPV is less than 10 in low-dimensional setting.

The results from simulation studies and illustration with real data revealed that while the MLE produced overfitted model with poor predictive performance (in terms of calibration), all penalized methods offered some improvements except for the RIDGE which in turn produced underfitted models (calibration slope greater than 1 with large variability). All other penalized methods (Firthtype and both $\log F(1,1)$ and $\log F(2,2))$ offered similar amount of improvement in calibration. However, the improvement in the discrimination in general was lower than that in calibration. The reason can be explained similarly with Pavlou et al. [10] as that the penalized methods tend to shrink the predicted probability towards the average compared with the MLE and hence the ordering of the predicted probabilities with and without experiencing the event in most patient pairs tends to remain unchanged after shringkage, which resulted in small improvement in AUC values of the penalized methods over MLE. All the penalized methods offered some improvement in the overall predictive performance (lower BS compared to those with MLE). Although all penalized methods correctly estimate the average predicted probability, Firthtype penalization introduced bias. The findings are similar to what obtained in other studies [10] that explored the use of some penalized methods such as ridge, lasso etc in risk predictions for low-dimensional data.

\section{Conclusions}

Based on the findings of the study it can be recommended to use $\log F$-type penalized method instead of MLE in risk prediction for low dimensional data small or sparse data. Because firstly this approach showed minimum bias in the estimate of regression coefficient and greater improvement in predictive performance than MLE, particularly in calibration by removing the amount of overfitting to some extents. Secondly, this approach has some additional advantage particularly for solving the problems due to separation. Of the two types of $\log F$ penalization, $\log F(1,1)$ is preferable to $\log F(2,2)$ because $\log F(2,2)$ though provides similar predictive performance but produces some bias in the regression coefficient particularly for the dichotomous covariates. Although the Firth-type penalized method have great advantage for solving the problems related to separation and showed comparable results with the $\log F$-type penalized methods with respect to calibration, discrimination and overall predictive performance, it produced bias in the estimate of the average predicted probability. The reason is that Firth's approach imposes prior on the intercept (which control the average predicted probability) and as a result it shrink the average predicted probability towards 0.5 and hence produced upward bias in the average predicted probability. However, the $\log F$ make the intercept free from the penalization and hence correctly estimates the 
Table 5 Performance of penalized methods in predicting cardiac events

\begin{tabular}{|c|c|c|c|c|}
\hline \multicolumn{5}{|c|}{ Models for predicting the risk of MI (EPV $\approx 7$ ) } \\
\hline Methods & Calibration Slope & AUC & Brier Score & APP \\
\hline MLE & $0.696(0.258)$ & $0.768(0.051)$ & 0.047 & 0.051 \\
\hline Firth & $0.706(0.260)$ & $0.766(0.052)$ & 0.049 & 0.057 \\
\hline $\log F(1,1)$ & $0.713(0.265)$ & $0.769(0.051)$ & 0.048 & 0.052 \\
\hline $\log F(2,2)$ & $0.723(0.271)$ & $0.769(0.051)$ & 0.048 & 0.052 \\
\hline RIDGE & $0.772(0.309)$ & $0.762(0.053)$ & 0.047 & 0.050 \\
\hline \multicolumn{5}{|c|}{ Models for predicting the risk of $C A B G(E P V \approx 10)$} \\
\hline MLE & $0.912(0.219)$ & $0.814(0.046)$ & 0.057 & 0.056 \\
\hline Firth & $0.909(0.217)$ & $0.814(0.046)$ & 0.056 & 0.059 \\
\hline $\log F(1,1)$ & $0.921(0.221)$ & $0.814(0.046)$ & 0.056 & 0.055 \\
\hline $\log F(2,2)$ & $0.926(0.223)$ & $0.813(0.046)$ & 0.057 & 0.055 \\
\hline RIDGE & $0.886(0.217)$ & $0.814(0.046)$ & 0.057 & 0.055 \\
\hline \multicolumn{5}{|c|}{ Models for predicting the risk of PTCA (EPV $\approx 5$ ) } \\
\hline MLE & $0.718(0.291)$ & $0.730(0.108)$ & 0.034 & 0.061 \\
\hline Firth & $0.721(0.279)$ & $0.729(0.108)$ & 0.035 & 0.066 \\
\hline $\log F(1,1)$ & $0.721(0.298)$ & $0.728(0.107)$ & 0.034 & 0.061 \\
\hline $\log F(2,2)$ & $0.720(0.305)$ & $0.728(0.107)$ & 0.034 & 0.061 \\
\hline RIDGE & $0.774(0.544)$ & $0.727(0.107)$ & 0.033 & 0.061 \\
\hline \multicolumn{5}{|c|}{ Models for predicting the risk of cardiac death $(E P V \approx 6)$} \\
\hline MLE & $0.661(0.529)$ & $0.688(0.121)$ & 0.024 & 0.062 \\
\hline Firth & $0.680(0.545)$ & $0.688(0.121)$ & 0.024 & 0.067 \\
\hline $\log F(1,1)$ & $0.645(0.535)$ & $0.687(0.120)$ & 0.024 & 0.062 \\
\hline $\log F(2,2)$ & $0.623(0.538)$ & $0.687(0.120)$ & 0.024 & 0.061 \\
\hline RIDGE & $0.665(0.608)$ & $0.684(0.121)$ & 0.023 & 0.062 \\
\hline \multicolumn{5}{|c|}{ Models for predicting the risk of any cardiac event $(E P V \approx 15)$} \\
\hline MLE & $0.942(0.206)$ & $0.771(0.044)$ & 0.059 & 0.164 \\
\hline Firth & $0.946(0.207)$ & $0.767(0.044)$ & 0.059 & 0.167 \\
\hline $\log F(1,1)$ & $0.945(0.206)$ & $0.770(0.044)$ & 0.058 & 0.164 \\
\hline $\log F(2,2)$ & $0.946(0.207)$ & $0.770(0.044)$ & 0.058 & 0.164 \\
\hline RIDGE & $1.004(0.222)$ & $0.769(0.044)$ & 0.056 & 0.165 \\
\hline
\end{tabular}

Event Per Variable (EPV) was calculated based on the number of event in training data. Estimates of the performance measures with SE in the parenthesis

average predicted probability. Similarly although RIDGE showed greater improvement in the discrimination and the overall predictive performance, it often provides under-fitted model. The striking disadvantages of RIDGE is that it has frequent convergence failure for data with low EPV or if there is separation. The rate was high (even higher than MLE) if data have combination of both low EPV and separation. This finding is similar to those [31] which reported low EPV or separation or combination of both as one of the reasons for the convergence-failure in RIDGE, although other studies [32] reported it as wrong choice (small value) of tuning parameter.
In the presence of separation, developing a risk model using any other penalized methods, except for the Firthtype and $\log F$-type methods, under study is challenging. Because RIDGE and LASSO-type penalized methods were originally developed particularly either for shrinking the regression coefficient or variable selection in high dimensional data rather than solving separation problem. However, the main limitation of $\log F$ type penalized approach is that it cannot be used directly for variable selection. If small-to moderate-level of variable selection is required in low-dimensional data with sufficient number of predictors, $\log F$ method can also be used 
in risk prediction after selecting important predictors using results from exploratory analysis of the data and likelihood ratio test conducted in different combinations of nested models.

This study did not focus on the use of Firth-type and $\log F$-type penalized method in risk prediction for lowdimensional survival data with few events where standard Cox regression is reported to be unreliable [33]. Further research may be possible to evaluate the predictive performance of these methods in comparison with the standard Cox model and the other penalized methods.

\section{Abbreviations}

APP: Average predicted probability; AUC: Area under receiver operating characteristic curve; EPV: Event per variable; FIRTH: Firth's penalized method; MLE: Maximum likelihood estimation

\section{Acknowledgements}

The authors acknowledge Alan Garfinkel and Frank Harrell for making available the dataset used in this study in a public domain under the department of biostatistics, Vanderbilt University, USA. In addition the authors thankful to associate editors and the reviewers for the valuabale suggestion and comments which strengthen the quality of the paper.

\section{Funding}

The authors received no specific fund for this study. They did this research based on their on interest and used data from secondary source.

\section{Availability of data and materials}

The dataset used in this study can be downloaded freely from a public domain at http://biostat.mc.vanderbilt.edu/DataSets under the authority of the department of biostatistics, Vanderbilt University, USA.

\section{Authors' contribution}

MSR contributed to the design of the study; MSR and MS analyzed the data; MSR and MS wrote the manuscript. All authors have read and approved the final manuscript.

\section{Competing interests}

The authors declared that they have no competing interest.

\section{Consent for publication}

Not applicable.

\section{Ethics approval and consent to participate}

As the dataset is freely available in a public domain at http://biostat.mc. vanderbilt.edu/DataSets and is permitted to use in research publication, the ethics approval and consent statement has been approved by the authority who made the data available for public use.

Received: 25 July 2016 Accepted: 16 February 2017

Published online: 23 February 2017

\section{References}

1. Abu-Hanna A, Lucas PJF. Prognostic models in medicine. Methods Inform Med. 2001;40:1-5

2. Moons KGM, Royston P, Vergouwe $Y$, Grobbee DE, Altman DG. Prognosis and prognostic research: what, why and how?. BMJ. 2009a;338:1317-20.

3. Altman DG, Royston P. What do you mean by validating a prognostic model?. Stat Med. 2000;19:453-73.

4. Moons KGM, Altman DG, Vergouwe Y, Royston P. Prognosis and prognostic research: application and impact of prognostic models in clinical practice. BMJ. 2009b;338:1487-90.

5. Peduzzi P, Concato J, Kemper E, Holford TR, Feinstein AR. A simulation study of the number of events per variable in logistic regression analysis. J Clin Epidemiol. 1996:49:1373-9.
6. Moons KG, de Groot JA, Linnet K, Reitsma JB, Bossuyt PM. Quantifying the added value of a diagnostic test or marker. Clin Chem. 2012;58(10): 1408-17.

7. Bouwmeester W, Zuithoff N, Mallett S, Geerlings MI, Vergouwe Y, Steyerberg EW, Altman DG, Moons KGM. Reporting and methods in clinical prediction research: a systematic review. PLOS Medecine. 2012;9(5):e1001221

8. Steyerberg EW, Eijkemans MJC, Harrell FE, Habbema JDF. Prognostic modelling with logistic regression analysis: a comparison of selection and estimation methods in small data sets. Stat Med. 2000;19(8):1059-79.

9. Ambler G, Seaman S, Omar RZ. An evaluation of penalised survival methods for developing prognostic models with rare events. Stat Med. 2012;31(11-12, SI):1150-61.

10. Pavlou M, Ambler G, Seaman S, De lorio M, RZ O. Review and evaluation of penalised regression methods for risk prediction in low-dimensional data with few events. Stat Med. 2016;35(7):1159-77.

11. Cessie SL, van Houwelingen JC. Ridge estimators in logistic regression. J R Stat Soc Series C. 1992;41(1):191-201.

12. Tibshirani R. Regression shrinkage and selection via the lasso. J R Stat Soc Series B. 1996;58:267-88.

13. Zou H, Hastie T. Regularization and variable selection via the elastic net. J R Stat Soc Series B. 2005;67(2):301-20.

14. Zou H. The adaptive lasso and its oracle properties. J Am Stat Assoc. 2006;101(476):1418-29.

15. Zou H, Zhang HH. On the adaptive elastic-net with a diverging number of parameters. Ann Stat. 2009;37(4):1733-51.

16. Albert A, Anderson JA. On the existence of maximum likelihood estimates in logistic regression models. Biometrika. 1984;71(1):1-10.

17. Heinze G, Scemper M. A solution to the problem of separation in logistic regression. Stat Med. 2002;21(16):2409-19.

18. Schaefer RL. Bias correction in maximum likelihood logistic regression. Stat Med. 1983;2:71-8.

19. Firth D. Bias reduction of maximum likelihood estimates. Biomertika. 1993:80:27-38.

20. Greenland S, Schwartzbaum JA, Finkle WD. Problems due to small samples and sparse data in conditional logistic regression analysis. Am J Epidemiol. 2000;151(5):531-9.

21. Lipsitz SR, Fitzmaurice G, Regenbogen SE, Sinha D, Ibrahim JG, Gawande AA. Bias correction for the proportional odds logistic regression model with application to a study of surgical complications. J R Stat Soc Series C. 2013:62(2):233-50.

22. Greenland S, Mansournia MA, Altman DG. Sparse data bias: a problem hiding in plain sight. BMJ. 2016:352:i1981.

23. Greenland S, Mansournia MA. Penalization, bias reduction, and default priors in logistic and related categorical and survival regressions. Stat Med. 2015;34(23):3133-43.

24. Greenland S. Prior data for non-normal priors. Stat Med. 2007;26:3578-90.

25. Greenland S. Generalized conjugate priors for bayesian analysis of risk and survival regressions. Biometrics. 2003;59:92-9.

26. Steyerberg EW, Vickers AJ, Cook NR, Gerds T, Gonen M, Obuchowski N, Pencina MJ, Kattan MW. Assessing the performance of prediction models: a framework for traditional and novel measures. Epidemiology. 2009;21(1):128-38.

27. Krivokapich J, Child J, Walter DO, Garfinkel A. Prognostic value of dobutamine stress echocardiography in predicting cardiac events in patients with known or suspected coronary artery disease. J Am Coll Cardiol. 1999;33(3):708-16.

28. Collins GS, Ogundimu EO, Altman DG. Sample size considerations for the external validation of a multivariable prognostic model: a resampling study. Stat Med. 2016:35(2):214-26.

29. Benner A, Zucknick M, Hielscher T, Ittrich C, Mansmann U. High-dimensional cox models: the choice of penalty as part of the model building process. Biometrical J. 2010;52:50-69.

30. van Wieringen WN, Kun D, Hampel AL R, Boulesteix. Survival prediction using gene expression data: a review and comparison. Comput Stat Data Anal. 2009:53:1590-603

31. Shen J, Gao S. A solution to separation and multicollinearity in multiple logistic regression. J Data Sci. 2008;6(4):515-31.

32. Goeman J, Meijer R, Chaturvedi N. L1 and L2 penalized regression models. R Package version 0.9-47; 2016. Retrieved from http://CRAN.R-project.org/ package $=$ penalized 
33. Ojeda FM, Müller C, D B, A TD, Schillert A, Heinig M, Zeller T, Schnabel RB. Comparison of cox model methods in a low-dimensional setting with few events. Genomics Proteomics Bioinforma. 2016;14(4):235-43.

Submit your next manuscript to BioMed Central and we will help you at every step:

- We accept pre-submission inquiries

- Our selector tool helps you to find the most relevant journal

- We provide round the clock customer support

- Convenient online submission

- Thorough peer review

- Inclusion in PubMed and all major indexing services

- Maximum visibility for your research

Submit your manuscript at www.biomedcentral.com/submit 